\title{
Uso de poda verde, plásticos refletivos, antitranspirante e potássio na produção de pêssegos
}

\author{
Renato Trevisan ${ }^{(1)}$, Flávio Gilberto Herter(1), Enilton Fick Coutinho( ${ }^{(1)}$, Emerson Dias Gonçalves ${ }^{(1)}$, \\ Carlos Augusto Posser Silveira ${ }^{(1)}$ e Cláudio José da Silva Freire ${ }^{(1)}$
}

(1)Embrapa Clima Temperado, Caixa Postal 403, CEP 96001-970 Pelotas RS. E-mail: trevisan@cpact.embrapa.br, herter@cpact.embrapa.br, enilton@cpact.embrapa.br, emersong@cpact.embrapa.br, guto@cpact.embrapa.br, freire@cpact.embrapa.br

Resumo - O objetivo deste trabalho foi avaliar técnicas que viabilizem a produção de pêssegos de melhor qualidade, ao potencializar características externas como a coloração vermelha na epiderme e o tamanho, bem como características químicas relativas ao sabor. Num pomar comercial de pessegueiros da cultivar Maciel, no Município de Pelotas, RS, foram avaliados os efeitos da poda verde, o uso de dois tipos de plástico refletivo sob a copa das plantas, diferentes concentrações de cloreto de potássio $(\mathrm{KCl})$ incorporado ao solo, com ou sem $\mathrm{KCl}$ via foliar e antitranspirante. $\mathrm{O}$ delineamento experimental utilizado foi o de blocos ao acaso. $\mathrm{O}$ uso de $1.200 \mathrm{~g}$ de $\mathrm{KCl}$ via solo, combinado com $10 \mathrm{~g}$ de $\mathrm{KCl}$ via foliar e poda verde, produziu coloração vermelha mais intensa na epiderme dos pêssegos. $\mathrm{O}$ uso de $1.600 \mathrm{~g}$ de $\mathrm{KCl}$ no solo, combinado com poda verde, produziu pêssegos com maior peso médio, maior diâmetro e baixa acidez titulável. A cobertura do solo, sob a copa das plantas, com plástico de ráfia, combinado com poda verde, resultou num maior teor de sólidos solúveis totais nos frutos. O uso dessas práticas culturais, realizadas na pré-colheita, influencia positivamente a qualidade final das frutas de pessegueiro da cultivar Maciel.

Termos para indexação: Prunus persica, coloração da epiderme, preferência do consumidor.

\section{Using green pruning, reflective plastics, antitranspirant and potassium in peach production}

\begin{abstract}
The objective of this work was to evaluate techniques which make possible the production of peaches of high quality by improving external characteristics, percent of red cover color, fruit size, and chemical characteristics that influence flavor, in a field peach of cultivar Maciel, in Pelotas, RS, Brazil. Effects evaluated were: vegetative pruning, the use of two types of reflective plastics under the plant canopy, the different potassium chloride concentrations incorporated to the soil, with and without foliar aplication of $\mathrm{KCl}$, and the use of antitranspirant. The experiment was carried out in a randomized block design. The results showed that the use of 1,200 $\mathrm{g}$ of $\mathrm{KCl}$ applied to soil, combined with $10 \mathrm{~g}$ of $\mathrm{KCl}$ as foliar application and vegetative pruning, provided intense red coloration of the peaches epidermis. The use of $1,600 \mathrm{~g}$ of $\mathrm{KCl}$ in the soil, combined with green pruning, resulted in higher average weight, larger diameter and low titratable acidity. Soil cover with raffia plastic under plant canopy, combined with green pruning, resulted in higher soluble solids content in the fruits. These practics during preharvest influence positively the final quality of cultivar Maciel peaches.
\end{abstract}

Index terms: Prunus persica, epidermis coloration, preference of consumer.

\section{Introdução}

O pessegueiro é uma das mais importantes espécies frutíferas de clima temperado exploradas no Brasil, que gera renda e divisas para o País. Na safra de 2003, foram produzidas $220.364 \mathrm{t}$, em 25.507 ha. O Rio Grande do Sul, nesse mesmo período, produziu aproximadamente 50\% da produção nacional, 112.005 t
(Pêssego..., 2006). A região de Pelotas, RS, possui uma área de produção significativa, com aproximadamente 8.000 ha, dos quais cerca de $95 \%$ são cultivados com pêssego para indústria (João et al., 2002). O reduzido porcentual destinado ao consumo in natura, 5\%, é fornecido ao consumidor com aparência visual de baixa qualidade (frutos pequenos, com defeitos e pouca coloração). 
Os principais aspectos exigidos no mercado de frutas, principalmente in natura, vinculados à qualidade, são a coloração da epiderme, cor da polpa, sabor, forma, firmeza, aroma, proteínas, bem como ausência de substâncias nocivas (Crisosto et al., 1997). Tal conceito envolve, além de características genéticas, os processos utilizados na produção, principalmente práticas culturais de manejo da planta e pomar.

Os principais fatores que determinam a qualidade das frutas na pré-colheita relacionam-se ao ambiente externo (temperatura, umidade relativa do ar, radiação solar, vento, altitude, quantidade de chuva e propriedades do solo), ao interno (cultivar, requerimento de fatores de produção próprios de cada cultivar, eficiência fotossintética) e manejo fitotécnico (nutrição mineral, manejo do solo, poda, raleio, reguladores de crescimento, densidade de plantio, irrigação e drenagem, entre outras) (Weston \& Barth, 1997).

A prática da poda verde em pessegueiro proporciona maior radiação solar e aeração no interior da planta e, conseqüentemente, intensifica a coloração vermelha na epiderme e melhora a sanidade dos frutos (Francisconi et al., 1996).

O uso de plásticos refletivos sobrepostos, sob a copa das plantas, é uma técnica de manejo muito utilizada em alguns países da América, Europa e Ásia. O objetivo principal é refletir a luz solar para o interior da copa, para intensificar a coloração vermelha da epiderme das frutas, melhorar o sabor, como também antecipar a maturação. Essa luz adicional é benéfica para a fotossíntese e produção de antocianinas (Layne et al., 2002). No Brasil, não há informações sobre o uso dessa técnica na cultura do pessegueiro.

O potássio (K) é encontrado em grande quantidade em todos os tecidos da planta. Atua na respiração, na síntese de carboidratos e proteínas e nas atividades enzimáticas. Níveis adequados de K são necessários para melhorar a qualidade das frutas, como a coloração vermelha na epiderme. Hunsche et al. (2003) observaram que diferentes doses de $\mathrm{K}$ proporcionaram incremento positivo na coloração vermelha em maçãs.

O uso de fitorreguladores, como auxinas, paclobutrazol, ethefon e antitraspirantes, melhoram as características de qualidade como a coloração das frutas, tamanho e sabor, pois promovem a atividade da enzima fenilalanina-amônia-liase (PAL), o que faz aumentar a síntese das antocianinas. (Li et al., 2002).

A utilização de práticas de manejo adequadas, com a adoção de tecnologias disponíveis, possibilitará aos fruticultores os recursos necessários para que se sobressaiam no mercado, ao produzir frutas com alta qualidade e competitividade comercial.

O objetivo deste trabalho foi estudar técnicas que viabilizem a produção de pêssegos de qualidade, com a potencialização de características externas como coloração vermelha e tamanho, bem como, características químicas relativas ao sabor.

\section{Material e Métodos}

O experimento foi instalado em setembro de 2002, em Pelotas, RS, num pomar comercial de pessegueiro da cultivar Maciel, com plantas de três anos de idade, conduzidas na forma de taça e espaçadas em 2x6 m.

O solo da área experimental foi classificado como Argissolo Vermelho-Amarelo distrófico típico (Embrapa, 1999). O delineamento experimental utilizado foi o de blocos ao acaso, com nove tratamentos e três repetições, com duas plantas úteis por parcela, isolada das demais por uma planta de bordadura.

O manejo fitotécnico do pomar e as demais práticas culturais foram realizados de acordo com o manejo convencional do produtor. Os tratamentos constaram de: 1) testemunha: manejo convencional do produtor, sem a realização da poda verde ou de qualquer outro tratamento; 2) poda verde: eliminação de ramos ladrões no interior da copa e base do tronco, quatro semanas antes da colheita; 3) plástico de ráfia mais poda verde: as áreas sob as plantas úteis foram cobertas, cinco semanas antes da colheita, com manta de plástico de ráfia $\left(16 \mathrm{~m}^{2}\right)$; 4) plástico metalizado mais poda verde: 0 procedimento de instalação e de montagem da cobertura de plástico sobre o solo foi idêntico ao do tratamento 3; 5) foram aplicados ao solo $800 \mathrm{~g}$ de $\mathrm{KCl}$ por planta, fracionados em quatro incorporações de $200 \mathrm{~g}$ por planta, com auxílio de enxada, numa profundidade em torno de $15 \mathrm{~cm}$, em intervalos de aproximadamente dez dias entre uma incorporação e outra, seguida da poda verde. Nas folhas, aplicaram-se $6 \mathrm{~g}$ de $\mathrm{KCl}$ por planta, fracionados em três pulverizações de $2 \mathrm{~g}$ por planta. As pulverizações foram realizadas nos mesmos dias das incorporações ao solo, com intervalos de dez dias; 6) foram aplicados ao solo $1.200 \mathrm{~g}$ de $\mathrm{KCl}$ por planta, fracionados em seis incorporações de 200 g por planta, com auxílio de enxada, numa profundidade em torno de $15 \mathrm{~cm}$, em intervalos de aproximadamente dez dias entre uma incorporação e outra, seguida de poda verde. 
Nas folhas, aplicaram-se $10 \mathrm{~g}$ de $\mathrm{KCl}$ por planta, fracionados em cinco pulverizações de $2 \mathrm{~g}$ por planta. As pulverizações foram realizadas nos mesmos dias das incorporações ao solo, com intervalos de dez dias; 7) foram aplicados ao solo $1.600 \mathrm{~g}$ de $\mathrm{KCl}$ por planta, fracionados em oito incorporações de 200 g por planta, com auxílio de enxada, numa profundidade em torno de $15 \mathrm{~cm}$, em intervalos de aproximadamente dez dias entre uma incorporação e outra, seguida da poda verde. Nas folhas, aplicaram-se $14 \mathrm{~g}$ de $\mathrm{KCl}$ por planta, fracionados em sete pulverizações de $2 \mathrm{~g}$ por planta. As pulverizações foram realizadas nos mesmos dias das incorporações ao solo, com intervalos de dez dias; 8) foram aplicados ao solo $1.600 \mathrm{~g}$ de $\mathrm{KCl}$ por planta, fracionados em oito incorporações de $200 \mathrm{~g}$ por planta, com auxílio de enxada, numa profundidade em torno de $15 \mathrm{~cm}$, em intervalos de aproximadamente dez dias entre uma incorporação e outra, seguida da poda verde e sem pulverização na folha; 9) poda verde e antitranspirante (Vapor Gard), pulverizado na concentração de $52 \mathrm{~mL}$ em $10 \mathrm{~L}$ de água, com pulverizador costal de capacidade de $20 \mathrm{~L}$. Nos tratamentos 3 a 9, a poda verde foi executada do mesmo modo usado no tratamento 2 , com eliminação dos ramos ladrões no interior das plantas e na base do tronco.

As aplicações de K tiveram início na primeira quinzena de setembro, e final na primeira quinzena de novembro de 2002. A poda verde, em todos os tratamentos, foi realizada 89 dias depois da plena floração.

A colheita, em todos os tratamentos, foi realizada em 17 de dezembro de 2002, com 15 frutas por tratamento para a análise das seguintes variáveis; cor da epiderme: mensurada com colorímetro eletrônico Minolta 300, iluminante D65 e abertura de $8 \mathrm{~mm}$. Registrado pela Commission Internationale de I' Eclaire (CIE) $\mathrm{L}^{*}, \mathrm{a}^{*}$ e $\mathrm{b}^{*}$, tendo-se usado as coordenadas espaciais de cor. O colorímetro, antes do início das leituras, foi calibrado sob cerâmica branca "stander". Neste sistema de representação de cor, os valores L*, $a^{*}$ e b* descrevem a uniformidade da cor no estado tridimensional, onde $\mathrm{L}^{*}$ corresponde ao escuro brilhoso (0, preto; 100 branco). Os valores de $\mathrm{a}^{*}$ correspondem à escala do verde ao vermelho ( $\mathrm{a}^{*}$ negativo, cor verde; $a *$ positivo, vermelho), e os valores de $b^{*}$ correspondem à escala do azul ao amarelo ( $\mathrm{b}^{*}$ negativo, cor azul; b* positivo, amarela). A partir desses valores, calculou-se a tonalidade da cor (ângulo $\mathrm{h}^{\circ}$ ) pela fórmula $h^{\circ}=\tan ^{-1} b^{*} / a^{*}$. O ângulo $h^{0}$ é a variável que melhor representa a evolução da cor na epiderme. Foram realizadas quatro leituras na região equatorial de cada fruta avaliada; peso médio das frutas, com balança digital; firmeza da polpa das frutas por meio de penetrômetro manual, com ponteira plana de $8 \mathrm{~mm}$ de diâmetro depois da remoção de uma pequena e superficial porção da casca, em lados opostos da região equatorial, foram efetuadas duas leituras por fruta, sendo os resultados expressos em Newton (N); teor de sólidos solúveis (SST) determinado no suco das frutas por refratometria, sendo os resultados expressos em ${ }^{\circ}$ Brix; acidez titulável total (ATT) com titulação potenciométrica com $\mathrm{NaOH} 0,1 \mathrm{~N}$ até $\mathrm{pH} 8,10$, de $10 \mathrm{~g}$ da amostra triturada em $90 \mathrm{~mL}$ de água destilada, sendo os resultados expressos em porcentagem de acidez.

Os dados foram submetidos à análise de variância, e as médias foram comparadas pelo teste de Duncan a $5 \%$ de probabilidade. As análises estatísticas foram processadas pelo programa WinStat (Machado \& Conceição, 2005).

\section{Resultados e Discussões}

A coloração da epiderme das frutas, em conseqüência dos tratamentos aplicados, está representada na Figura 1. De acordo com os pigmentos predominantes e sua distribuição, as frutas apresentam cor de fundo, que corresponde fundamentalmente às clorofilas e carotenóides, nos tons verde, amarelo e alaranjado, e cor de superfície determinada pelas antocianinas, cuja síntese resulta na coloração vermelha (Chitarra \& Chitarra, 2005). Com o avanço da maturação, a coloração verde diminui e aumenta a cor amarela. Isso ocorre em razão da degradação das clorofilas e ativação das clorofilases (Ihl et al., 1994).

A intensidade da coloração vermelha foi maior na epiderme das frutas do tratamento 6 , embora não tenha diferido significativamente dos tratamentos 3 , 7 e 8 (Tabela 1). O mecanismo pelo qual o $\mathrm{K}$ influencia o aumento da coloração vermelha na epiderme, não está bem esclarecido, porém, não se pode descartar a hipótese de que atue como cofator para enzimas específicas da formação dos pigmentos. Esses resultados corroboram os de Hunsche et al. (2003), que observaram aumento da coloração vermelha na epiderme de maçãs, quando se forneceu $\mathrm{K}$ para as plantas.

Com o uso de material refletivo (tratamento 3), também se pôde observar maior intensidade de coloração vermelha na epiderme das frutas (Tabela 1). Esses resultados são semelhantes aos descritos por Layne et al. 
(2001, 2002), que observaram aumentos entre 16 e $46 \%$ da coloração vermelha na epiderme de pêssegos e maçãs, quando utilizaram material refletivo sob a copa das plantas.

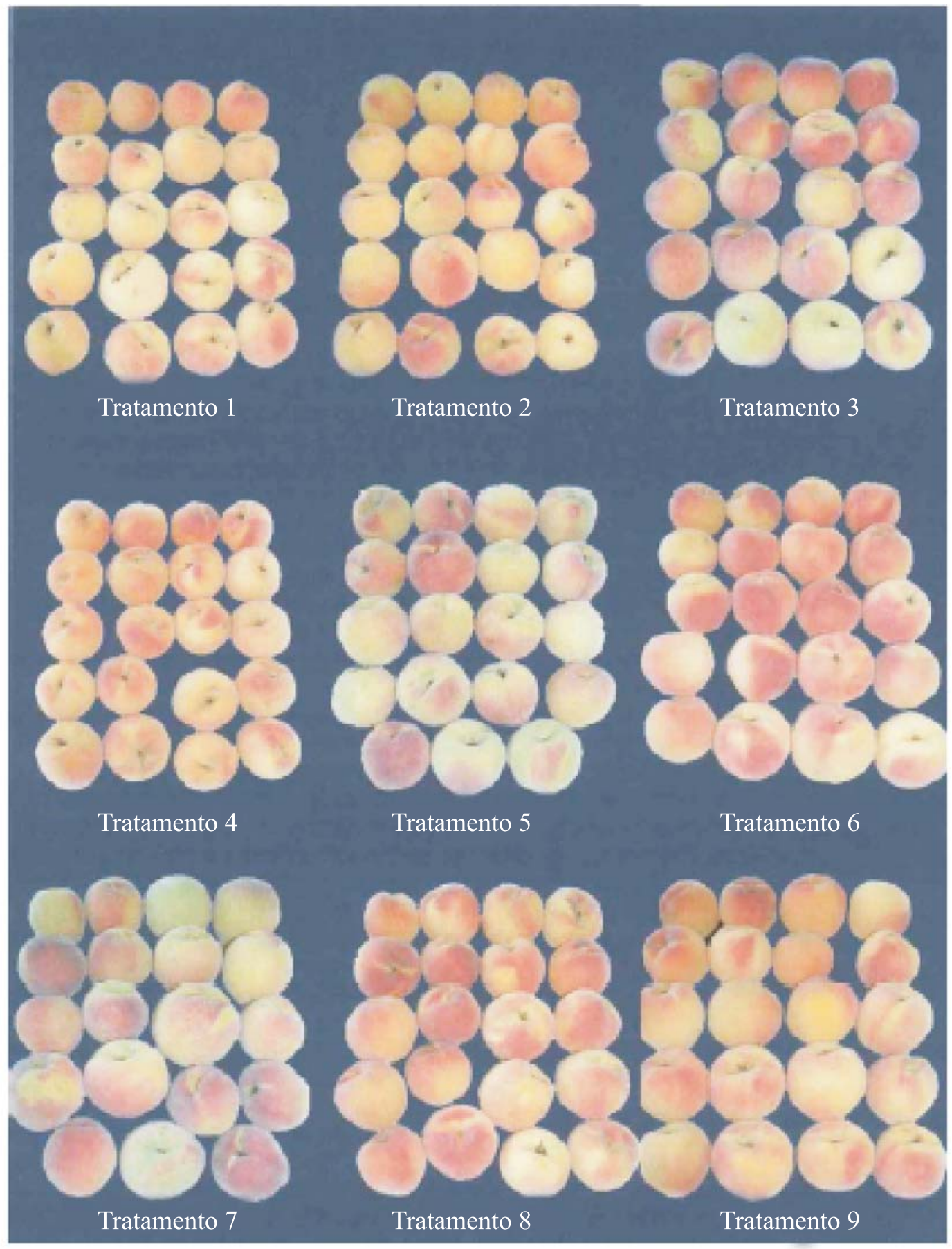

Figura 1. Coloração da epiderme de pêssegos, cultivar Maciel, em conseqüência dos tratamentos: 1) testemunha; 2) poda verde; 3) plástico de ráfia e poda verde; 4) plástico metalizado e poda verde; 5) 800 g de $\mathrm{KCl}$ no solo + 3 aplicações de 2 g de $\mathrm{KCl}$ foliar e poda verde; 6) 1.200 g de KCl no solo + 5 aplicações de 2 g de $\mathrm{KCl}$ foliar e poda verde; 7) $1.600 \mathrm{~g}$ de $\mathrm{KCl}$ no solo + 7 aplicações de $2 \mathrm{~g}$ de $\mathrm{KCl}$ foliar e poda verde; 8) $1.600 \mathrm{~g}$ de $\mathrm{KCl}$ no solo e poda verde; 9) antitranspirante e poda verde. 
Miller \& Greene (2003) observaram diferenças significativas na coloração da epiderme de maçãs, ao utilizar diferentes materiais refletivos, plástico branco e metalizado, o que não foi constatado neste trabalho, em que o uso de plástico de ráfia e plástico metalizado não diferiu significativamente, embora tenha diferido da testemunha.

Os plásticos, ao refletir a luz solar, aumentam a síntese da enzima UFGalT (Ju et al., 1999) e refletem os raios ultravioleta, o que aumenta a atividade da fenil amônia liase (Wilson, 1994), intensificando, desta forma, a coloração vermelha da epiderme. Neste contexto, pode haver duas maneiras de a luz solar aumentar a síntese das antocianinas e o seu acúmulo nas frutas. A primeira é com aumento da atividade fotossintética da planta, pelo provimento de substratos para a fruta e, assim, indiretamente estimular a síntese das antocianinas pelas substâncias de reserva. A outra, é a ação direta dos plásticos em estimular a síntese de antocianinas.

O peso de frutos foi maior no tratamento 8, embora não tenha diferido estatisticamente dos tratamentos 5, 6 e 7 , todos com aplicação de $\mathrm{K}$ no solo e na folha. O mesmo fato foi observado para o diâmetro das frutas (Tabela 1). Segundo Stiles (1994), o K induz a formação de um vacúolo central grande, que regula a entrada da água nas células e ativa várias enzimas envolvidas na fotossíntese e na respiração, proporcionando assim maior diâmetro às frutas. Hunsche et al. (2003) atribuíram o maior diâmetro em maçãs ao uso de maiores doses de K. Comparando-se o diâmetro das frutas obtidas nos tratamentos 7 e 8, supõe-se que as aplicações foliares desse nutriente não tiveram efeito sobre este parâmetro.

A perda da firmeza de polpa, de modo geral, ocorre em razão de seu amaciamento pelo amadurecimento normal dos frutos, que é um processo complexo e envolve diferentes mecanismos (Chitarra \& Chitarra, 2005). O tratamento 2 (poda verde) determinou maior firmeza de polpa, não tendo diferido dos tratamentos 5 e 6. O tratamento 4 , apresentou menor firmeza, não diferindo dos tratamentos 1, 7 e 8 (Tabela 1).

Miller (1987), ao realizar poda verde em pêssegos da cultivar Loring, também observou aumento da resistência da polpa. Porém, Gerhardt et al. (1991) e Francisconi et al. (1996) realizaram poda verde nas cultivares Premier e Marli e não observaram diferenças significativas. As menores doses de $\mathrm{K}$ (tratamentos 5 e 6) também determinaram maior firmeza de polpa, enquanto as maiores doses proporcionaram seu amaciamento. O mesmo fato foi observado em maçãs com o aumento das doses de K (Hunsch et al., 2003).

O teor de sólidos solúveis totais fornece o indicativo da quantidade de açúcares presentes nas frutas. Observa-se que no tratamento 3 (plástico de ráfia mais poda verde) ocorreu uma produção de frutas com ${ }^{\circ}$ Brix significativamente superior ao obtido nos tratamentos 1 , 5, 6, 7, 8 e 9, não tendo diferido dos tratamentos 2 e 4 (Tabela 1). Layne et al. (2001) encontraram pequenas diferenças no teor de açúcares nas cultivares Loring, Summer Gold, Bounty, Sunprince Cresthaven e Encore. Ao contrário, Layne \& Rushing (1999) não observaram alterações no teor de açúcares, quando disponibilizaram

Tabela 1. Coloração da epiderme (ângulo Hue), peso médio dos frutos, diâmetro médio dos frutos, firmeza da polpa, sólidos solúveis totais (SST) e acidez titulável total (ATT) de pêssegos, cultivar Maciel, em conseqüência dos tratamentos aplicados(1).

\begin{tabular}{|c|c|c|c|c|c|c|}
\hline Tratamento & $\begin{array}{l}\text { Ângulo } \\
\text { Hue }^{(2)}\end{array}$ & $\begin{array}{c}\text { Peso } \\
(\mathrm{g}) \\
\end{array}$ & $\begin{array}{c}\text { Diâmetro } \\
(\mathrm{mm})\end{array}$ & $\begin{array}{l}\text { Firmeza } \\
\left(\mathrm{lb} \mathrm{pol}^{-2}\right)\end{array}$ & $\begin{array}{c}\text { SST } \\
\left({ }^{\circ} \text { Brix }\right)\end{array}$ & $\begin{array}{l}\text { ATT } \\
(\%) \\
\end{array}$ \\
\hline 1. Testemunha & $87,1 \mathrm{a}$ & $124,4 b$ & $56,6 \mathrm{c}$ & $9,8 \mathrm{~cd}$ & $11,33 b$ & $0,74 b c$ \\
\hline 2. Poda verde & $83,3 \mathrm{ab}$ & $112,4 b$ & $61,1 b$ & $12,2 \mathrm{a}$ & $11,60 \mathrm{ab}$ & $0,76 \mathrm{ab}$ \\
\hline 3. Plástico de ráfia e poda verde & $72,8 \mathrm{~cd}$ & $113,2 b$ & $61,6 b$ & $11,3 b$ & $11,67 \mathrm{a}$ & $0,72 \mathrm{ab}$ \\
\hline 4. Plástico metalizado e poda verde & $77,1 \mathrm{bc}$ & $110,7 \mathrm{~b}$ & $62,7 \mathrm{~b}$ & $9,4 d$ & $11,53 \mathrm{ab}$ & $0,64 \mathrm{~d}$ \\
\hline 5. $800 \mathrm{~g}$ de $\mathrm{KCl}$ solo $+3 \mathrm{x}$ de $2 \mathrm{~g}$ de $\mathrm{KCl}$ foliar e poda verde & $77,6 \mathrm{bc}$ & $124,0 \mathrm{ab}$ & $63,2 b$ & $11,4 \mathrm{ab}$ & $10,73 \mathrm{bc}$ & $0,79 \mathrm{a}$ \\
\hline 6. $1.200 \mathrm{~g}$ de $\mathrm{KCl}$ solo $+5 \mathrm{x}$ de $2 \mathrm{~g}$ de $\mathrm{KCl}$ foliar e poda verde & $61,2 \mathrm{~d}$ & $125,6 \mathrm{ab}$ & $61,7 \mathrm{~b}$ & $11,5 \mathrm{ab}$ & $10,40 \mathrm{c}$ & $0,70 \mathrm{bc}$ \\
\hline 7. $1.600 \mathrm{~g}$ de $\mathrm{KCl}$ solo $+7 \mathrm{x}$ de $2 \mathrm{~g}$ de $\mathrm{KCl}$ foliar e poda verde & $73,3 \mathrm{~cd}$ & $123,8 \mathrm{ab}$ & $63,7 \mathrm{~b}$ & $10,3 \mathrm{~cd}$ & $10,07 \mathrm{c}$ & $0,76 \mathrm{ab}$ \\
\hline 8. $1.600 \mathrm{~g}$ de $\mathrm{KCl}$ solo e poda verde & $74,3 \mathrm{~cd}$ & $129,2 \mathrm{a}$ & $68,5 \mathrm{a}$ & $10,2 \mathrm{~cd}$ & $10,40 \mathrm{c}$ & $0,68 \mathrm{~cd}$ \\
\hline 9. Antitranspirante (Vapor gard) e poda verde & $77,6 \mathrm{bc}$ & $112,1 \mathrm{~b}$ & $63,0 \mathrm{~b}$ & $10,6 \mathrm{bc}$ & $10,67 \mathrm{bc}$ & $0,71 \mathrm{bc}$ \\
\hline Média & 77,0 & 118,1 & 62,5 & 4,7 & 10,94 & 0,72 \\
\hline $\mathrm{CV}(\%)$ & 4,70 & 6,46 & 2,90 & 9,80 & 4,60 & 5,35 \\
\hline
\end{tabular}

${ }^{(1)}$ Médias seguidas de letras iguais, na coluna, não diferem entre si pelo teste de Duncan a $5 \%$ de probabilidade. (2) Ângulo Hue* $=\tan ^{-1} \mathrm{~b}^{*} / \mathrm{a}^{*}\left(0^{\circ}=\right.$ vermelho, $90^{\circ}=\mathrm{amarelo}$, $180^{\circ}=$ verde). 
material refletivo sob a copa de plantas de pessegueiro, cultivares Cresthaven e Encore.

A acidez dos frutos, que pode aumentar ou diminuir entre uma safra e outra, é influenciada por condições climáticas, estádio de maturação e localização da fruta na planta. $\mathrm{O}$ tratamento 5 , embora não tenha diferido significativamente dos tratamentos 2, 3 e 7, ocasionou maior porcentual de acidez aos frutos. Jaeger \& Putter (1999) relataram que o K fornecido às plantas proporciona maior acidez às frutas, fato também observado neste trabalho. Hunsch et al. (2003) também observaram que com o uso de doses crescentes de $\mathrm{K}$ a acidez das frutas aumenta.

\section{Conclusões}

1. Frutos de pessegueiro, cultivar Maciel, de película com coloração vermelha mais intensa e de maior peso médio e diâmetro, são obtidos com o uso de cloreto de potássio e poda verde.

2. Plástico de ráfia e poda verde proporcionam maior teor de sólidos solúveis totais, e plástico metalizado e poda verde proporcionam menor acidez nos frutos de pessegueiro da cultivar Maciel.

\section{Referências}

CHITARRA, M.I.F.; CHITARRA, A.B. Pós-colheita de frutas e hortaliças: fisiologia e manejo. 2.ed. Lavras: Ufla, 2005. 785p.

CRISOSTO, C.H.; JOHNSON, R.S.; DeJONG, T.; DAY, K.R. Orchard factors affecting postharvest stone fruit quality. HortScience, v.32, p.820-823, 1997.

EMBRAPA. Centro Nacional de Pesquisa de Solos (Rio de Janeiro, RJ). Sistema brasileiro de classificação de solos. Brasília: Embrapa-SPI; Rio de Janeiro: Embrapa-CNPS, 1999. 412p.

FRANCISCONI, A.H.D.; BARRADAS, C.I.N.; MARODIN, G.A.B. Efeito da poda verde na qualidade do fruto e na produção de pessegueiro cv. Marli. Pesquisa Agropecuária Brasileira, v.31, p.51-54, 1996.

GERHARDT, I.R.; BARRADAS, C.I.N.; MARODIN, G.A.B. Efeito de tipos e épocas de poda verde sobre a qualidade e produção das frutas de pessegueiro (Prunus persica) (L.) Batsch) "Premier". Revista Brasileira de Fruticultura, v.13, p.177-181, 1991.

HUNSCHE, M.; BRACKMANN, A.; EMANI, P.R. Efeito da adubação potássica na qualidade pós-colheita de maçãs
Fuji. Pesquisa Agropecuária Brasileira, v.38, p.489-496, 2003.

IHL, M.; ETCHEBERRIGARAY, C.; BIFANI, C. Chlorophyllase behavior on "Granny Smith” apples. Acta Horticulturae, v.368, p.58-68, 1994.

JAEGER, A.; PUTTER, H. Preharvest factors and postharvest quality decline of apples. Acta Horticulturae, v.485, p.103-110, 1999.

JOÃO, L.P.; ROSA, J.I. da; FERRI, V.C.; MARTINELLO, M.D. Levantamento da fruticultura comercial do Rio Grande do Sul. Porto Alegre: Emater-RS/Ascar, 2002. p.55-58.

JU, Z.; DUAN, Y.; JU, Z. Effects of covering the orchardfloor with reflecting films on pigment accumulation and fruit coloration in 'Fuji' apples. Scientia Horticulturae, v.82, p.47-56, 1999.

LAYNE, D.R.; JIANG, Z.; RUSHING, J.W. The influence of reflective film and ReTain on red skin coloration and maturity of Gala apples. HortTechnology, v.12, p.640-644, 2002.

LAYNE, D.R.; JIANG, Z.; RUSHING, J.W. Tree fruit reflective film improves red skin coloration and advances maturity in peach. HortTechnology, v.11, p.234-242, 2001.

LAYNE, D.R.; RUSHING, J.W. Color sells: reflective film may improve color and quality in your peaches and apples. Fruit Grower, v.119, p.18-19, 1999.

LI, Z.H.; GEMMA, H.; IWAHORI, S. Stimulation of 'Fuji' apple skin color by ethephon and phosphorus-calcium mixed compounds in relation to flavonoid synthesis. Scientia Horticulturae, v.94, p.193-199, 2002.

MACHADO, A.A.; CONCEIÇÃO, A.R. WinStat: sistema de análise estatística para Windows. Versão Beta. Pelotas: Universidade Federal de Pelotas, 2005. Não paginado.

MILLER, S.S. Summer pruning affects fruit quality of peach as influenced by dormant pruning, summer pruning and summer topping, HortScience, v.22, p.390-393, 1987.

MILLER, S.S.; GREENE, G.M. The use of reflective film and ethephon to improve red skin color of apples in the Mid-Atlantic region of the United States. HortTecnology, v.13, p.90-99, 2003.

PÊSSEGO: produção brasileira. In: AGRIANUAL 2006. São Paulo: FNP, 2006. p.418-424.

STILES, W.C. Phosphorus, potassium, magnesium, and sulfur soil management. In: PETERSON, B.; STEVENS, R.G. (Ed.). Tree fruit nutrition: a comprehensive manual of deciduous tree fruit nutrient needs. Yakima: Good Fruit Grower, 1994. p.63-70.

WESTON, L.A.; BARTH, M.M. Preharvest factors affecting postharvest quality of vegetables. HortScience, v.32, p.812-816, 1997.

WILSON, R. Potential of induced resistance to control postharvest diseases of fruits and vegetables. Plant Disease, v.18, p.837-844, 1994.

Recebido em 4 de julho de 2005 e aprovado em 26 de maio de 2006 